\title{
Management of Bladder Pain Syndrome (BPS): A Practical Guide
}

\author{
Patrick Juliebø-Jones $\mathbb{D},{ }^{1,2}$ Karin M. Hjelle, ${ }^{1,2}$ Jannike Mohn $\mathbb{D},^{1}$ \\ Gigja Gudbrandsdottir $\mathbb{D}^{\mathbb{1}},{ }^{1,2}$ Ingunn Roth, ${ }^{1}$ Adeel Asghar Chaudhry, \\ Anne Kvåle Bergesen, ${ }^{1}$ and Christian Beisland ${ }^{10}{ }^{1,2}$ \\ ${ }^{1}$ Department of Urology, Haukeland University Hospital, Bergen, Norway
${ }^{2}$ Department of Clinical Medicine, University of Bergen, Bergen, Norway \\ Correspondence should be addressed to Patrick Juliebø-Jones; jonesurology@gmail.com
}

Received 19 November 2021; Accepted 20 December 2021; Published 10 January 2022

Academic Editor: Miroslav Djordjevic

Copyright (C) 2022 Patrick Juliebø-Jones et al. This is an open access article distributed under the Creative Commons Attribution License, which permits unrestricted use, distribution, and reproduction in any medium, provided the original work is properly cited.

\begin{abstract}
Bladder pain syndrome (BPS) is a prevalent and pervasive disease. The physical and psychological sequelae can be very burdensome for the patient, and the condition represents a real challenge for the clinician as well. With no simple pathognomonic test, finding harmony in navigating patient care can be demanding. Diagnosis and management rely upon a multidisciplinary and holistic approach. Treatment options include conservative measures and pharmacotherapies as well as bladder instillation therapies. Ultimately, surgery may be offered but only in cases of refractory disease. This article offers a pragmatic guide for clinicians managing this challenging disease.
\end{abstract}

\section{Manuscript}

Bladder pain syndrome (BPS) is a disease of ubiquity, and epidemiological studies estimate it to affect $1.8-51 / 10,000$ individuals worldwide [1]. However, there is evidence to suggest that the prevalence is underreported and that less than $10 \%$ of disease sufferers receive a formal diagnosis [2]. Prior to recent changes in standardised terminology, BPS was formerly referred to as interstitial cystitis (IC) [3]. This shift in nomenclature reflects its multifactorial aetiology and the symptom-based approach, which now occupies the diagnostic workup. The burden of the BPS is substantial, regarding both physical and psychological sequelae. It has been highlighted by the World Health Organization (WHO) as a major public health issue $[4,5]$. Difficulties in diagnosing the condition are mirrored by the challenges faced in treating it. Despite the availability of an abundance of novel treatments, most of these have a limited evidence basis to support their use [6]. Such is the diversity in clinical phenotypes associated with BPS, and there is a huge range in treatment strategies offered worldwide. While numerous international guidelines do exist, recommendations can be divergent $[7,8]$. It can therefore be quite difficult for the clinician to find harmony in navigating patient care. Our aim was to provide an overview of a practical framework for the contemporary management of BPS.

\section{Methods}

The diagnostic and treatment pathways outlined here have been constructed based on a comprehensive review of world literature. The following bibliographic databases were searched: MEDLINE, Scopus, and CINAHL. The search terms included but were not limited to "bladder pain syndrome," "interstitial cystitis," and "treatment." International guidelines and the chronic care model (CCM) were also evaluated $[9,11]$. The approach outlined is multidisciplinary and includes urologists, specialist nurses (urotherapists), and gynaecologists with an extended network to involve pain specialists, primary care physicians (PCPs), and psychologists.

2.1. The Challenge of BPS. The first step for clinicians involved in the care of BPS is an appreciation and understanding of why it is such a challenging condition to treat. From uncertainty regarding pathophysiological causation, disagreement in disease definition, heterogeneity in clinical 
phenotypes, to the lack of pathognomonic investigations and limitations in treatment efficacy, each part is contentious and renders BPS to hold an enigmatic status. Transparency and honesty with the patient about this from the outset are recommended to manage and set realistic expectations. Yeh et al. recorded outcomes of BPS patients at long-term followup (mean duration 16.6 \pm 9.75 years) [12]. Only $12 \%$ achieved a status of being symptom-free, and $47 \%$ reported an improvement of at least $50 \%$ compared with the baseline. In a small number (6\%), their condition continued to worsen despite treatment. Therefore, patients need to be counselled carefully and in a sensitive manner. The patients should be advised that they will be embarking on a journey, which can be both long and frustrating. More than one-third of patients receive at least four different treatment types. Such are the frustrations associated with having a "pain that cannot be seen," and many patients with BPS disengage with medical care despite having ongoing symptoms [13]. The multifactorial nature of BPS demands a management pathway, which reflects this. A dedicated multidisciplinary team (MDT) meeting is recommended to discuss patients as required. The patients can be reassured that a range of healthcare professionals will be involved in their care [10]. A survey of PCPs' knowledge of BPS revealed that only $61 \%$ correctly answered that it was not caused by a psychiatric illness [14]. Consultations for BPS generally demand more time and so it is worth scheduling the clinic time to be longer than normal if possible.

2.2. Presentation. While BPS can affect males, up to $90 \%$ of sufferers are females [15]. Berry et al. determined that females diagnosed with BPS are more likely to be multiparous and unmarried [2]. Over $90 \%$ of patients with BPS are Caucasian, and the average age at diagnosis is the fourth decade of life [10]. The delay from symptom onset to diagnosis is common and is estimated to be 3 to 7 years [16]. Medical records of patients with BPS reveal a significantly higher number of visits to healthcare providers [4]. The International Continence Society (ICS) defines BPS as "persistent or recurrent chronic pelvic pain, pressure or discomfort perceived to be related to the urinary bladder accompanied by at least one other urinary symptom such as an urgent need to void or urinary frequency" [17]. Several other definitions exist; however, common to all of them are themes of chronicity, pelvic pain, and accompanying bothersome lower urinary tract symptoms (LUTS) [10]. Regarding the latter, urinary frequency, urgency, and nocturia are the most commonly reported. Ito et al. reported that the urinary frequency was reported in $98.3 \%$ of patients [18]. Typically, the urinary frequency associated with BPS ranges from 8 to 50 times a day. While urinary urgency is common, urinary incontinence (UI) is not. Urinary urgency associated with BPS is driven by the principal need to void in order to stop pain rather than to prevent leakage [8]. Pain is a hallmark symptom, and indeed, BPS is now classed as a subdivision of the chronic pelvic pain umbrella according to the European Association of Urology (EAU) guidelines [7]. The patients may report pain in all parts of the pelvic region, and it may be associated with triggers such as specific foods/beverages and/or sexual intercourse. The patients may have been previously managed with several courses of antibiotics in the community but to no avail. The constellation of burdensome symptoms associated with BPS results in an estimated $42 \%$ of patients not working and $18 \%$ of patients only working part time [19]. The studies investigating the natural history of BPS reveal that the symptom burden progressively worsens over a period of 3 to 5 years and reaches a plateau thereafter [20]. Late deterioration of symptoms is rare [10]. The psychological impact is pervasive, and patients are likely to have concomitant anxiety (14-52\%) and/or depression (16-70\%) [20].

\subsection{Diagnosis}

2.3.1. History. Historically, diagnostic criteria such as those developed by the National Institute of Diabetes and Digestive and Kidney Diseases (NIDDK) included objective stigmata such as cystoscopic confirmation of diffuse glomerulations or Hunner's lesions (HLs) as well as certain cystometric properties such as a bladder capacity of less than $350 \mathrm{ml}$ when awake [21]. A paradigm shift has taken place towards a diagnosis established on the presence of the abovementioned symptom constellation (Table 1). A detailed history is therefore required to elicit the symptomatology. Regarding family history, twin studies reveal that there is likely a genetic component to BPS, but this remains poorly understood [22].

2.3.2. Examination. All patients should undergo a physical examination. This should include the abdomen and external genitalia as well as bimanual pelvic examination in women. Strength, tone, and point tenderness of pelvic floor muscles should be documented [6]. Urologists may be less familiar with the intricacies of this particular examination, and additional training from gynaecology colleagues can be valuable. Focused neurological examination in all patients to rule out occult disease should be performed, as well as a digital rectal examination (DRE) in men [23].

2.3.3. Useful Adjuncts. Such is the difficulty associated with the bladder being "an unreliable witness," and supplementation of the history through validated questionnaires is beneficial $[24,25]$. The symptom areas are highlighted, which are burdening the patient the most, and they offer a means of grading severity. They also serve as a baseline assessment for comparison after treatment. There are many patient-reported outcome measures (PROMs) used in the setting of BPS including the O'Leary-Sant interstitial cystitis symptom index (ICSI), Bladder Pain/Interstitial Cystitis Symptom Score (BPIC-SS), and Pain, Urgency, Frequency (PUF) score. There are also several relevant modules from the International Consultation on Incontinence Questionnaires (ICIQs) such as Female Lower Urinary Tract 
TABLE 1: Characteristics of BPS.

\begin{tabular}{|c|c|c|c|}
\hline Risk factors & $\begin{array}{l}\text { Presenting } \\
\text { symptoms }\end{array}$ & Diagnoses to exclude & $\begin{array}{l}\text { Common coexisting } \\
\text { conditions }\end{array}$ \\
\hline $\begin{array}{l}\text { Female gender } \\
\text { Age } \\
\text { Depression } \\
\text { Smoking history } \\
\text { High intake caffeine } \\
\text { Lower socioeconomic } \\
\text { Group } \\
\text { Multiparity } \\
\text { Lower socioeconomic } \\
\text { group }\end{array}$ & $\begin{array}{c}\text { Core: } \\
\text { Pain } \\
\text { Frequency } \\
\text { Urgency } \\
\text { N.B chronic } \\
\text { duration. } \\
\text { Additional: } \\
\text { Nocturia } \\
\text { Dyspareunia } \\
\text { Anal discomfort }\end{array}$ & $\begin{array}{c}\text { Males and females: } \\
\text { Urinary tract infection } \\
\text { Malignancy } \\
\text { Urinary stone disease } \\
\text { Overactive bladder } \\
\text { Pelvic adhesions } \\
\text { Inflammatory bowel disease } \\
\text { Hernia } \\
\text { Genital herpes } \\
\text { Cystitis caused by radiation, tuberculosis, or chemicals, e.g., } \\
\text { cyclophosphamide } \\
\text { Ketamine bladder } \\
\text { Pudendal neuropathy } \\
\text { Multiple sclerosis } \\
\text { Spinal cord injury } \\
\text { Diverticular disease (of the bowel) } \\
\text { Females: } \\
\text { Endometriosis } \\
\text { Pelvic inflammatory disease } \\
\text { Vaginitis } \\
\text { Eroded mesh } \\
\text { Urethral diverticulum } \\
\text { Urogenital prolapse } \\
\text { Pregnancy } \\
\text { Pelvic floor muscle tightness } \\
\text { Males: } \\
\text { Chronic prostatitis } \\
\text { Bladder outflow obstruction }\end{array}$ & $\begin{array}{c}\text { Fibromyalgia } \\
\text { Irritable bowel syndrome } \\
\text { Vulvodynia } \\
\text { Sjogren's syndrome } \\
\text { Chronic headache } \\
\text { Chronic fatigue syndrome } \\
\text { Depression } \\
\text { Anxiety }\end{array}$ \\
\hline
\end{tabular}

Symptoms (ICIQ-FLUTS) and Lower Urinary Tract Symptoms Quality of Life Module (ICIQ-LUTSqol), which are available in several languages [26]. A bladder diary can also be issued to the patient to complete. It is worthwhile for centres to consider assembling a package of such assessment tools, which can be distributed to the patient in advance of the consultation [27].

\subsection{Investigations}

2.4.1. Simple Tests. On arrival to the clinic, patients should undergo urine dipstick (+/- culture) analysis and uroflowmetry including measurement of post-void residual (PVR) urine (Figure 1). Performing a urine culture can sometimes be considered in patients with negative dipstick to measure for a clinically significant bacteria burden, which may not reach standard thresholds for diagnosis $[27,28]$. Communication with a local microbiologist is recommended to explore this service. Those with relevant risk factors such as a history of smoking should be considered for urine cytology testing. The patient's electronic records should be studied to reveal the results of any previous urine cultures and recent blood tests. If the history has revealed any red flag symptoms such as visible haematuria or abnormal prostate on DRE, patients should be diverted to the appropriate oncological pathway.
2.4.2. Special Tests. While no single investigation can confirm a diagnosis, there are several additional tests, which can be considered. These can help support the diagnosis of BPS and rule out other conditions. Flexible cystoscopy under local anaesthetic (LA) can be performed to do the latter. However, rigid cystoscopy under general anaesthetic (GA) is needed to measure maximum bladder capacity and perform hydrodistension to observe for glomerulations. While glomerulations were previously considered an automatic criterion for BPS, this is no longer the case, as more recent research has revealed that glomerulations can also be present in healthy, asymptomatic individuals. For diagnosing BPS, a previous meta-analysis by Wennevik et al. revealed that glomerulations only carry a sensitivity and specificity of $60 \%$ and $62 \%$, respectively [29]. Cystoscopy may also reveal HLs, which typically display a central pale scar with a stellate appearance and are surrounded by reddened mucosa [30]. Biopsy shows characteristic inflammation with lymphoplasmacytic cells and increased vascularity and epithelial denudation [31]. The presence of HLs is also no longer a prerequisite for BPS diagnosis, and findings from the IC Database study showed that only $11.7 \%$ of women had HLs [32]. However, this endoscopic finding does categorise the patient into a specific phenotype termed "Ulcer-type" BPS [31]. Urodynamic studies (UDS) can be considered to help distinguish from other disorders such as overactive bladder (OAB), especially where patients have shown no response to first-line treatments. Kim et al. determined patients with BPS 


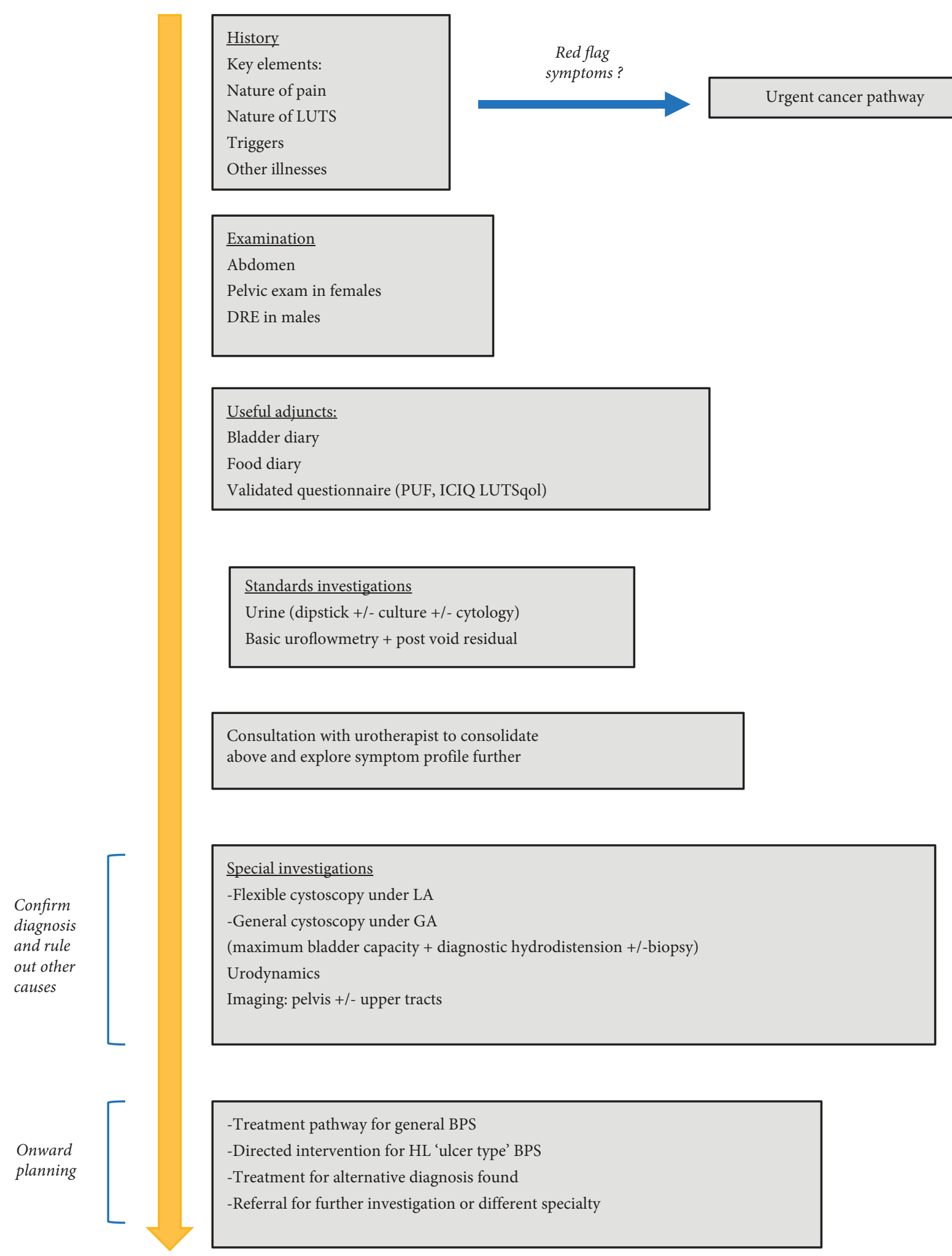

FIgURE 1: Diagnostic pathway for BPS.

to have an earlier first sensation of filling and a smaller cystometric capacity compared with OAB subjects, which is consistent with previous research [33]. UDS can also help investigate bladder outflow obstruction and poor detrusor contractility [34]. Of note, bladder filling during UDS in the context of BPS can be particularly uncomfortable for the patient. Pelvic and/or upper tract imaging can be considered by the clinician if it will help guide the diagnosis. 
2.4.3. Diagnostic Tests No Longer Part of Clinical Practice. In accordance with most guidelines, the authors do not perform the potassium sensitivity test as it can be extremely uncomfortable for the patient and even trigger symptom flares. In addition, the predictive value is low [7]. Intravesical anaesthetic challenge test with alkalinised lidocaine is no longer part of common clinical practice as it is supported by limited evidence [6]. Urinary biomarkers are a promising area of research in precision medicine but are not yet in clinical use [35].

2.4.4. Differential Diagnosis. There are many alternative diagnoses to consider during the investigation (Table 1). As reflected by the changes in terminology for BPS, central to the diagnosis of BPS is excluding other causes. Both the history and many of the investigations help serve to eliminate these alternatives. In all patients, a priority is to exclude a urological malignancy. Benign urological conditions to consider include urinary tract infection (UTI) and stone disease. Gastrointestinal symptoms should prompt the clinician to consider conditions such as diverticular disease and inflammatory bowel disease (IBD). While less common, the possibility of a neurological cause, e.g., multiple sclerosis, should not be overlooked. Certain conditions are genderspecific. In females, this includes gynaecological pathologies such as endometriosis and pelvic inflammatory disease (PID). In males, chronic prostatitis and bladder outflow obstruction are differential diagnoses.

\subsection{Treatment}

2.5.1. Initial. A pivotal approach to BPS is the early involvement of an urotherapist who can play a key diagnostic and therapeutic role [36]. In the Scandinavian setting, urotherapists are specialist nurses with a universityaccredited qualification in bladder dysfunction. It allows the patient's history to be explored in a supportive environment but also one of great experience in this area. A conservative treatment approach is always adopted first, and the establishment of realistic expectations is entered into early with the patient. Lifestyle modifications should be recommended such as the promotion of regular exercise and dietary modifications including reduction in caffeine intake and other common aggressors such as spicy foods, alcohol, and citrus fruits. This should be combined with counselling regarding smoking cessation. The patient's existing pain management strategies need to be addressed, and it is not uncommon for BPS patients to have adopted self-initiated remedies such as nonprescribed medication(s). The use of illicit drugs should prompt the clinician to rule out a possible diagnosis of ketamine cystitis. The cases of particularly complex pain should be considered for early referral to a specialist pain team. Written guidance can be provided to the PCP in case of future symptom flares in the community. This may be particularly useful where patients live in remote geographic locations. Several of the instillation therapies are now able to be self-administered at home.
Involvement of the patient's partner/family can be helpful during the consultation. Research shows that this can help sufferers deal with the disease [37]. Such is the diverse and often misleading information that is freely available to patients reading online about BPS, and written information about BPS can be provided to aid patient education. BPS can be lonely for the patient to experience and so the patient can be informed of support groups. These groups also often provide information on alternative and complementary therapies such as acupuncture, reflexology, and hypnotherapy. Symptoms can be worsened by stress, and therefore, exploring behavioural modifications can be of benefit and consideration for a talking therapy such as cognitive behavioural therapy (CBT). This can be facilitated by the PCP. Where the physical examination has elicited pelvic floor tenderness or trigger points, manual physical therapy can be initiated and the involvement of physiotherapist with relevant expertise may be warranted.

2.5.2. Oral Treatments. Oral pharmacotherapy forms the mainstay of the next step where conservative approaches have failed (Figure 2). Nonsteroidal anti-inflammatory drugs (NSAIDs) are the standard analgesia to begin with (and regular gastric protection) together with paracetamol. Tricyclic antidepressants (TCAs) such as amitriptyline are another option for treating pain and helping to ameliorate the burden of storage LUTS. Cimetidine, a histamine H2 receptor antagonist, can also be offered. This takes effect owing to a similar peptidergic pathway in the bladder [38].

2.5.3. Instillation Treatments. There are a number of instillation therapies available worldwide including chondroitin sulfate (CS), hyaluronic acid (HA), heparin, lidocaine, pentosan polysulfate sodium (PPS), and dimethyl sulfoxide (DMSO). The majority of these represent replenishment strategies to restore the architecture of the glycosaminoglycan (GAG) layer and the natural protection it provides [39]. Their clinical implementation is largely dependent on the licensing it receives in a particular country. In the European setting, chondroitin sulfate (CS) and hyaluronic acid (HA) are two of the most commonly used agents. A typical regime is once weekly for six weeks and monthly thereafter as required. HA and CS are also available as a combination (iAluRil ${ }^{\circledR}$ ). The commonest adverse events (AEs) are pain, irritation, and UTI. There is no strong evidence to recommend the superiority of any particular instillation therapy [39]. However, PPS and DMSO demonstrate worse side effect profiles (including headache and dizziness) and the need for ophthalmologic surveillance due to the risk of lens opacification [4].

2.5.4. Intradetrusor Injection of Botulinum Toxin Type A (BoNT-A). BoNT-A is an established treatment option for detrusor overactivity; however, it can also be considered in patients exhibiting a poor response to instillation treatments [41]. The standard initial dose is 100 units, which is consistent with most trials [41]. Cystoscopy (LA or GA) is required to deliver and inject the agent at approximately 


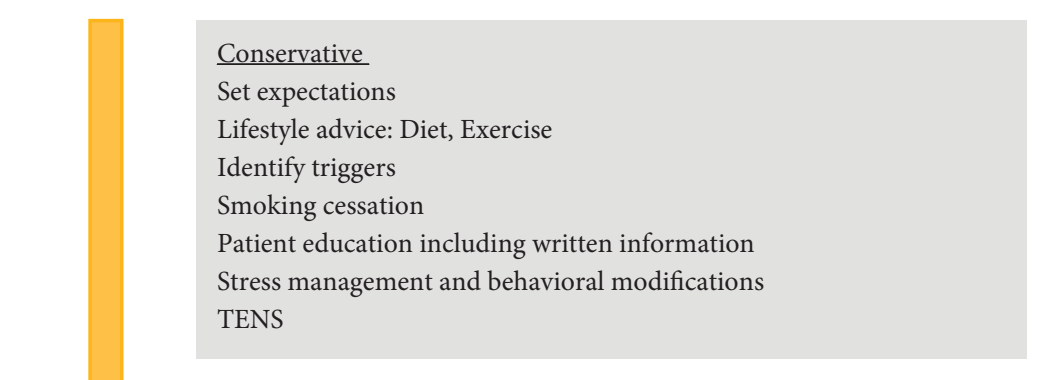

\section{Signposting:}

-Complementary therapies e.g., hypnotherapy,

acupuncture, reflexology

-Cognitive behavioral therapy (CBT)

-Support groups

Relevant referral to

-Physiotherapy for manual physical therapy

-Pain specialist

Pharmacotherapy:

-Pain relief $1^{\text {st }}$ line Paracetamol + NSAID (+ gastric protection)

$2^{\text {nd }}$ line TCA e.g., amitriptyline

-Histamine $\mathrm{H} 2$ receptor antagonist e.g., Cimetidine

-Oral PPS (if available in country)

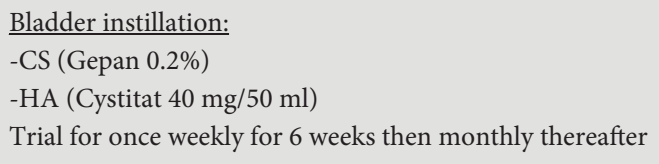

Major surgery e.g., urinary diversion with ileal conduit

(thorough patient counselling required)

FIgURe 2: Treatment pathway for BPS.

10-20 sites. Patients are administered an initial dose, and maintenance can be given according to response. The patients must be counselled regarding the risk of urinary retention and need to learn self-catheterisation.

2.5.5. Nerve Stimulation. Neuromodulators such as transcutaneous electrical nerve stimulation (TENS) work based on the gate control theory [42]. Simultaneous inputs are provided in large, myelinated nerve fibers, which serve to eliminate painful stimuli. TENS is a noninvasive and external device, which can be applied by the patient at home. This is a conservative treatment option. More invasive options are percutaneous tibial nerve stimulation (PTNS), pudendal nerve stimulation (PNS), and sacral nerve stimulation (SNS). These should only be considered in the later stages of treatment. These are available as permanent implants and are suitable options for patients with a good 
response to treatment with a temporary device. While success rates as high as $80 \%$ have been reported, only limited evidence exists to support the application of such implants for BPS [6]. However, careful counselling is required regarding AEs such as infection and the need for revision or removal. Both BoNT-A and invasive neuromodulators are categorised as fourth-line treatments in international guidelines; however, there is no recommendation beyond this to guide, which of these treatments should be tried first. However, BoNT-A is arguably the most natural next step to begin with after the other intravesical treatments have been trialed. On a practical level, it is also easier to offer this service compared with neuromodulation.

2.5.6. Oral Cyclosporine A. Oral cyclosporine A, an immunosuppressive agent, is now available as a possible treatment option for patients with refractory disease [43]. Fastidious monitoring is required for AEs such as hypertension and renal failure. A clinically significant proportion of patients will discontinue treatment early due to these sequelae [6-9].

2.5.7. Surgery. In those patients who have exhausted all other treatment options and whose symptoms significantly affect their quality of life, surgery can be considered. However, the decision for surgery requires careful patient counselling regarding morbidity (complications include infection, bowel obstruction, ureteric stricture, and stoma problems) and mortality associated with the surgery. It must also be explained that there is no guarantee that surgery will relieve their pain. Other long-term considerations for the patient include impact on sexual function, body image, and lifestyle [44]. The patient must understand that they are potentially swapping one set of problems for another. Options include urinary diversion with ileal conduit or a continent urinary diversion. The decision for whether a continent urinary diversion is opted for is influenced by factors such as patient preference and history of previous surgery and irradiation [45]. Urinary diversion can be done with or without cystectomy. However, there exists controversy surrounding whether it is permissible to leave the "diseased organ" in situ. As highlighted by the ICS, it appears that for many patients, the result of their native bladder no longer storing urine is sufficient to yield satisfactory symptom improvement. Pyocystis is reported to occur between 3.3 and $67 \%$ of cases [46].

\subsubsection{Interventions for HLs, “Ulcer-Type" BPS.} Endoscopic identification of HLs should prompt a directed treatment strategy. The removal of the scarred urothelial tissue can be achieved through transurethral resection (TUR) and coagulation/fulguration. In a series of 59 patients, $13.1 \%$ and $57.2 \%$ required repeat intervention at 12 months and 24 months, respectively [47]. Triamcinolone acetonide (TA), a long-acting synthetic steroid, can be endoscopically injected into the lesion [48]. This allows direct administration of the steroid compared to treatment with oral steroids, which is systemically absorbed. The latter is not recommended due to AEs such as hypertension and diabetes mellitus. Evidence is largely limited to small case series such as that by Funaro et al. [49]. Measuring pain using a Likert scale (0-10), it improved significantly at one-month followup ( 8.3 vs. $3, p<0.01)$ and the average time before retreatment was 345 days.

2.5.9. Treatments Not Recommended. Several treatments are no longer recommended in contemporary clinical practice. This includes intravesical Bacillus Calmette-Guerin (BCG), which is not used given that the side effects outweigh any potential therapeutic gains [9]. Long-term antibiotics are not advocated due to a lack of proven efficacy, side effects, and antimicrobial resistance (AMR). Long-duration ( $>10$ mins) and high-pressure ( $>80$ to $100 \mathrm{~cm} \mathrm{H}_{2}$ ) bladder distension has not been shown to improve outcomes and can be associated with serious complications such as bladder perforation [6].

\section{The Role of the Urologist}

Given the multidisciplinary approach, which is recommended in the management of BPS, the urologist is often in a unique position to act as the vehicle to coordinate such efforts and establish a local network of health professionals with relevant expertise. This is enhanced if individual departments can develop a treatment algorithm, which is tailored to the services they can offer. Having a nominated urologist to champion and lead such an initiative can complement this. Where local services for treatment services do not exist, e.g., urinary diversion or nerve stimulation, developing a referral pathway to a specialist centre is recommended. Maintaining communication with primary care teams is also valuable to update referral criteria, support for patient care, and provide educational events.

\section{Limitations and Future Research}

This review does not serve as an exhaustive guide to BPS management. However, it does offer pragmatic recommendations for this clinical challenge. Randomised studies are warranted to strengthen the evidence basis for many of the treatments and development of a universally agreed core outcome set [50].

\section{Conclusion}

BPS is a complex condition and is challenging for clinicians to manage. Diagnosis in the current era is established on the basis of clinical symptoms rather than pathognomonic criteria. Treatment follows a stepwise and multimodal approach. Extirpative surgery is offered but only in cases of refractory disease and must be supported by thorough preoperative counselling.

\section{Data Availability}

No data were used to support this study. 


\section{Conflicts of Interest}

The authors declare that they have no conflicts of interest.

\section{References}

[1] L. Nasta, "The prevalence of bladder pain syndrome/interstitial cystitis in Italy: the importance of a national registry," in Bladder Pain Syndrome-an Evolution, P. Hanno, J. Nordling, D. Staskin, A. Wein, and J. Wyndaele, Eds., Springer, Cham, Switzerland, 2018.

[2] S. H. Berry, M. N. Elliott, M. Suttorp et al., "Prevalence of symptoms of bladder pain syndrome/interstitial cystitis among adult females in the United States," The Journal of Urology, vol. 186, no. 2, pp. 540-544, 2011.

[3] R. Doggweiler, K. E. Whitmore, J. M. Meijlink et al., "A standard for terminology in chronic pelvic pain syndromes: a report from the chronic pelvic pain working group of the international continence society," Neurourology and Urodynamics, vol. 36, no. 4, pp. 984-1008, 2017.

[4] Z. Hakimi, J. Houbiers, R. Pedersini, and J. Vietri, "The burden of bladder pain in five European countries: a crosssectional study," Urology, vol. 99, pp. 84-91, 2017.

[5] World Health Organization, 1st International Consultation on Incontinence, Plymbridge Distributors, Plymouth, England, 1999.

[6] P. M. Hanno, D. Erickson, R. Moldwin, M. M. Faraday, and American Urological Association, "Diagnosis and treatment of interstitial cystitis/bladder pain syndrome: AUA guideline amendment," The Journal of Urology, vol. 193, no. 5, pp. 1545-1553, 2015.

[7] D. Engeler, A. P. Baranowski, J. Borovicka et al., EAU Guidelines on Chronic Pelvic Pain, EAU Guidelines Office, Arnhem, The Netherlands, 2018, http://uroweb.org/wpcontent/uploads/EAU-Guidelines-on-Chronic-Pelvic-Pain-20 18-large-text.pdf.

[8] S. A. Tirlapur, J. V. Birch, C. L. Carberry et al., "Irving A on behalf of the royal college of obstetricians and gynaecologists. Management of bladder pain syndrome," BJOG, vol. 124, pp. e46-e72, 2016.

[9] J. Mohn, K. Førde, and H. M. Lando, "NO2-03 bladder pain syndrome (BPS)-a retrospective cross-sectional survey of a hospital population (2010-2018) with focus on demographic characteristics and intravesical treatment modalities," $A b$ stracts, Scandinavian Journal of Urology, vol. 53, no. 221, pp. 1-38, 2019.

[10] T. Bodenheimer, E. H. Wagner, and K. Grumbach, "Improving primary care for patients with chronic illness," Journal of the American Medical Association, vol. 288, no. 14, pp. 1775-1779, 2002.

[11] J. Mohn, K. Førde, and H. M. Lando, "NO2-04, Bladder pain syndrome (BPS)-a glance at the complexity of the patient group in light of international guidelines and the Chronic Care Model," Abstracts, Scandinavian Journal of Urology, vol. 53, no. 221, pp. 1-38, 2019.

[12] H. L. Yeh, J. F. Jhang, Y. C. Kuo, and H. C. Kuo, "Long-term outcome and symptom improvement in patients with interstitial cystitis/bladder pain syndrome with or without regular follow-up and treatment," Neurourology and Urodynamics, vol. 38, no. 7, pp. 1985-1993, 2019.

[13] L. McGowan, K. Luker, F. Creed, and C. A. Chew-Graham, "How do you explain a pain that can't be seen?: the narratives of women with chronic pelvic pain and their disengagement with the diagnostic cycle," British Journal of Health Psychology, vol. 12, no. 2, pp. 261-274, 2007.

[14] J. Q. Clemens, E. A. Calhoun, M. S. Litwin et al., "A survey of primary care physician practices in the diagnosis and management of women with interstitial cystitis/painful bladder syndrome," Urology, vol. 76, no. 2, pp. 323-328, 2010.

[15] H. C. Arora and D. A. Shoskes, "The enigma of men with interstitial cystitis/bladder pain syndrome," Translational Andrology and Urology, vol. 4, no. 6, pp. 668-676, 2015.

[16] G. C. Curhan, F. E. Speizer, D. J. Hunter, S. G. Curhan, and M. J. Stampfer, "Epidemiology of interstitial cystitis: a population based study," The Journal of Urology, vol. 161, no. 2, pp. 549-552, 1999.

[17] 2021, https://www.ics.org/committees/standardisation/ terminologydiscussions/icbps.

[18] T. Ito, T. Ueda, Y. Honma, and M. Takei, "Recent trends in patient characteristics and therapeutic choices for interstitial cystitis: analysis of 282 Japanese patients," International Journal of Urology, vol. 14, no. 12, pp. 1068-1070, 2007.

[19] M. K. Beckett, M. N. Elliott, J. Q. Clemens, B. Ewing, and S. H. Berry, "Consequences of interstitial cystitis/bladder pain symptoms on women's work participation and income: results from a national household sample," The Journal of Urology, vol. 191, no. 1, pp. 83-88, 2014.

[20] L. C. McKernan, C. G. Walsh, W. S. Reynolds, L. J. Crofford, R. R. Dmochowski, and D. A. Williams, "Psychosocial comorbidities in Interstitial Cystitis/Bladder Pain syndrome (IC/BPS): a systematic review," Neurourology and Urodynamics, vol. 37, no. 3, pp. 926-941, 2018.

[21] P. M. Hanno, "Interstitial cystitis-epidemiology, diagnostic criteria, clinical markers," Reviews in Urology, vol. 4, no. Suppl 1, pp. S3-S8, 2002.

[22] E. Tunitsky, M. D. Barber, P. C. Jeppson, B. Nutter, J. E. Jelovsek, and B. Ridgeway, "Bladder pain syndrome/ interstitial cystitis in twin sisters," The Journal of Urology, vol. 187 , no. 1 , pp. 148-152, 2012.

[23] E. L. Chrysanthopoulou and S. K. Doumouchtsis, "Challenges and current evidence on the management of bladder pain syndrome," Neurourology and Urodynamics, vol. 33, no. 8, pp. 1193-1201, 2014.

[24] R. Turner-Warwick, C. G. Whiteside, P. H. L. Worth, E. J. G. Milroy, and C. P. Bates, "A urodynamic view of the clinical problems associated with bladder neck dysfunction and its treatment by endoscopic incision and trans-trigonal posterior Prostatectomy1," British Journal of Urology, vol. 45, no. 1, pp. 44-59, 1973.

[25] P. Abrams, A. P. Smith, and N. Cotterill, "The impact of urinary incontinence on health-related quality of life (HRQoL) in a real-world population of women aged 45-60 years: results from a survey in France, Germany, the UK and the USA," BJU International, vol. 115, no. 1, pp. 143-152, 2015.

[26] P. Abrams, K. Avery, N. Gardener, J. Donovan, and ICIQ Advisory Board, "The international consultation on incontinence modular questionnaire:," The Journal of Urology, vol. 175, no. 3 Pt 1, pp. 1063-1066, 2006, http://www.iciq.net.

[27] A. Cox, "Management of interstitial cystitis/bladder pain syndrome," Canadian Urological Association Journal, vol. 12, no. 6S3, pp. S157-S160, 2018.

[28] S. Heytens, A. De Sutter, L. Coorevits et al., "Women with symptoms of a urinary tract infection but a negative urine culture: PCR-based quantification of Escherichia coli suggests infection in most cases," Clinical Microbiology and Infections, vol. 23, no. 9, pp. 647-652, 2017. 
[29] G. E. Wennevik, J. M. Meijlink, P. Hanno, and J. Nordling, "The role of glomerulations in bladder pain syndrome: a review," The Journal of Urology, vol. 195, no. 1, pp. 19-25, 2016.

[30] K. E. Whitmore, M. Fall, A. Sengiku, H. Tomoe, Y. Logadottir, and Y. H. Kim, "Hunner lesion versus non-Hunner lesion interstitial cystitis/bladder pain syndrome," International Journal of Urology, vol. 26, no. 1, pp. 26-34, 2019.

[31] Y. Homma, "Interstitial cystitis, bladder pain syndrome, hypersensitive bladder, and interstitial cystitis/bladder pain syndrome-clarification of definitions and relationships," International Journal of Urology, vol. 26, no. 1, pp. 20-24, 2019.

[32] E. Messing, D. Pauk, A. Schaeffer et al., "Associations among cystoscopic findings and symptoms and physical examination findings in women enrolled in the Interstitial Cystitis Data Base (ICDB) Study," Urology, vol. 49, no. Suppl 5A, pp. 81-85, 1997.

[33] S. H. Kim, T. B. Kim, S. W. Kim, and S.-J. Oh, "Urodynamic findings of the painful bladder syndrome/interstitial cystitis: a comparison with idiopathic overactive bladder," The Journal of Urology, vol. 181, no. 6, pp. 2550-2554, 2009.

[34] Y.-C. Kuo and H.-C. Kuo, "The role of urodynamic study in evaluation of interstitial cystitis/painful bladder syndrome," Tzu Chi Medical Journal, vol. 25, no. 3, pp. 131-134, 2013.

[35] J. Kim, W. T. Kim, and W.-J. Kim, "Advances in urinary biomarker discovery in urological research," Investigative and Clinical Urology, vol. 61, no. Suppl 1, pp. S8-S22, 2020.

[36] V. Geissbuehler, S. Forst, M. Werner, C.-A. Schoenenberger, R. Berner, and C. Betschart, "Urotherapist activities in caring for patients with pelvic floor disorders: a prospective singlecenter observational study," Archives of Gynecology and Obstetrics, vol. 303, no. 2, pp. 471-479, 2021.

[37] A. Lahat, S. Neuman, R. Eliakim, and S. Ben-Horin, "Partners of patients with inflammatory bowel disease: how important is their support?" Clinical and Experimental Gastroenterology, vol. 7, pp. 255-259, 2014.

[38] P. Dasgupta, S. D. Sharma, C. Womack, H. N. Blackford, and P. Dennis, "Cimetidine in painful bladder syndrome: a histopathological study," BJU International, vol. 88, no. 3, pp. 183-186, 2001.

[39] P. Jones, K. M. Hjelle, J. Mohn et al., "Current status of intravesical therapies for bladder pain syndrome (BPS): a narrative review of emerging evidence," Urology, vol. 156, pp. e48-e57, 2021.

[40] D. Wang, A. Au, F. Gunnemann et al., "Pentosan-associated maculopathy: prevalence, screening guidelines, and spectrum of findings based on prospective multimodal analysis," $\mathrm{Ca}$ nadian Journal of Ophthalmology, vol. 55, no. 2, pp. 116-125, 2020.

[41] H.-C. Kuo, Y.-H. Jiang, Y.-C. Tsai, and Y.-C. Kuo, "Intravesical botulinum toxin-A injections reduce bladder pain of interstitial cystitis/bladder pain syndrome refractory to conventional treatment-a prospective, multicenter, randomized, double-blind, placebo-controlled clinical trial," Neurourology and Urodynamics, vol. 35, no. 5, pp. 609-614, 2016.

[42] N. Sharma, K. Rekha, and J. Srinivasan, "Efficacy of transcutaneous electrical nerve stimulation in the treatment of chronic pelvic pain," Journal of Mid-Life Health, vol. 8, no. 1, pp. 36-39, 2017.

[43] Z. Wang and L. Zhang, "Treatment effect of cyclosporine A in patients with painful bladder syndrome/interstitial cystitis: a systematic review," Experimental and Therapeutic Medicine, vol. 12, no. 1, pp. 445-450, 2016.
[44] N. I. Osman, D. G. Bratt, A. P. Downey, F. Esperto, R. D. Inman, and C. R. Chapple, "A systematic review of surgical interventions for the treatment of bladder pain syndrome/interstitial cystitis," European Urology Focus, vol. 7, no. 4, pp. 877-885, 2021.

[45] N. Vasdev, A. Thorpe, and A. Moon, "Continent urinary diversion," Indian Journal of Urology, vol. 29, no. 4, pp. 303-309, 2013.

[46] M. H. Kamel, R. Gardner, A. Tourchi et al., "Pyocystis: a systematic review," International Urology and Nephrology, vol. 49, no. 6, pp. 917-926, 2017.

[47] J. H. Hillelsohn, S. Rais-Bahrami, J. I. Friedlander et al., "Fulguration for Hunner ulcers: long-term clinical outcomes," The Journal of Urology, vol. 188, no. 6, pp. 2238-2241, 2012.

[48] M. Cox, J. J. Klutke, and C. G. Klutke, "Assessment of patient outcomes following submucosal injection of triamcinolone for treatment of Hunner's ulcer subtype interstitial cystitis," The Canadian Journal of Urology, vol. 16, pp. 4536-4540, 2009.

[49] M. G. Funaro, A. N. King, J. N. H. Stern, R. M. Moldwin, and S. Bahlani, "Endoscopic injection of low dose triamcinolone: a simple, minimally invasive, and effective therapy for interstitial cystitis with hunner lesions," Urology, vol. 118, pp. 25-29, 2018.

[50] D. C. Turk, R. H. Dworkin, R. R. Allen et al., "Core outcome domains for chronic pain clinical trials: IMMPACT recommendations," Pain, vol. 106, no. 3, pp. 337-345, 2003. 